\title{
Which mapping technique for population density is effective, attractive, and suggestive?
}

\author{
David Forrest $^{\mathrm{a}}{ }^{*}$, Beata Medyńska-Gulij ${ }^{\mathrm{b}}$ \\ ${ }^{a}$ University of Glasgow, School of Geographical \& Earth Sciences, United Kingdom, David.Forrest@glasgow.ac.uk \\ ${ }^{b}$ Adam Mickiewicz University Poznan, Department of Cartography and Geomatics, Poland, bmg@amu.edu.pl , \\ * Corresponding author: David Forrest, David.Forrest@glasgow.ac.uk
}

Keywords: graphical attractiveness, suggestive presentation, effectiveness of cartographic visualisations, mapping techniques, Central Europe, people density

\begin{abstract}
:
The effectiveness of cartographic visualisations is connected with the creation of maps that enable the simple and unequivocal reading of features of geographical phenomena. The effectiveness of visualisation is defined as both the efficiency (speed) and accuracy (correctness) of the communication of information. The graphical attractiveness of cartographic visualisations is connected with the individual, subjective feeling of readers concerning the subjective impressions of the beauty, aesthetics and originality of the cartographic presentation. The attractiveness of cartographic design encourages people to use maps and has a significant impact on shaping cartographic communication and the comprehensible quality of the map. Mapping methods vary in terms of effectiveness and graphical attractiveness. In many cases, an increase in graphical complexity improves the attractiveness of a visualisation, but frequently leads to a decrease in effectiveness. Two-dimensional methods are more effective than their three-dimensional counterparts, because in treedimensional views physical distances are subject to distortions ensuing from the perspective projection. The utilisation of textual labels can help to increase effectiveness by confirming locations and reducing reliance on the user's mental map of the area.
\end{abstract}
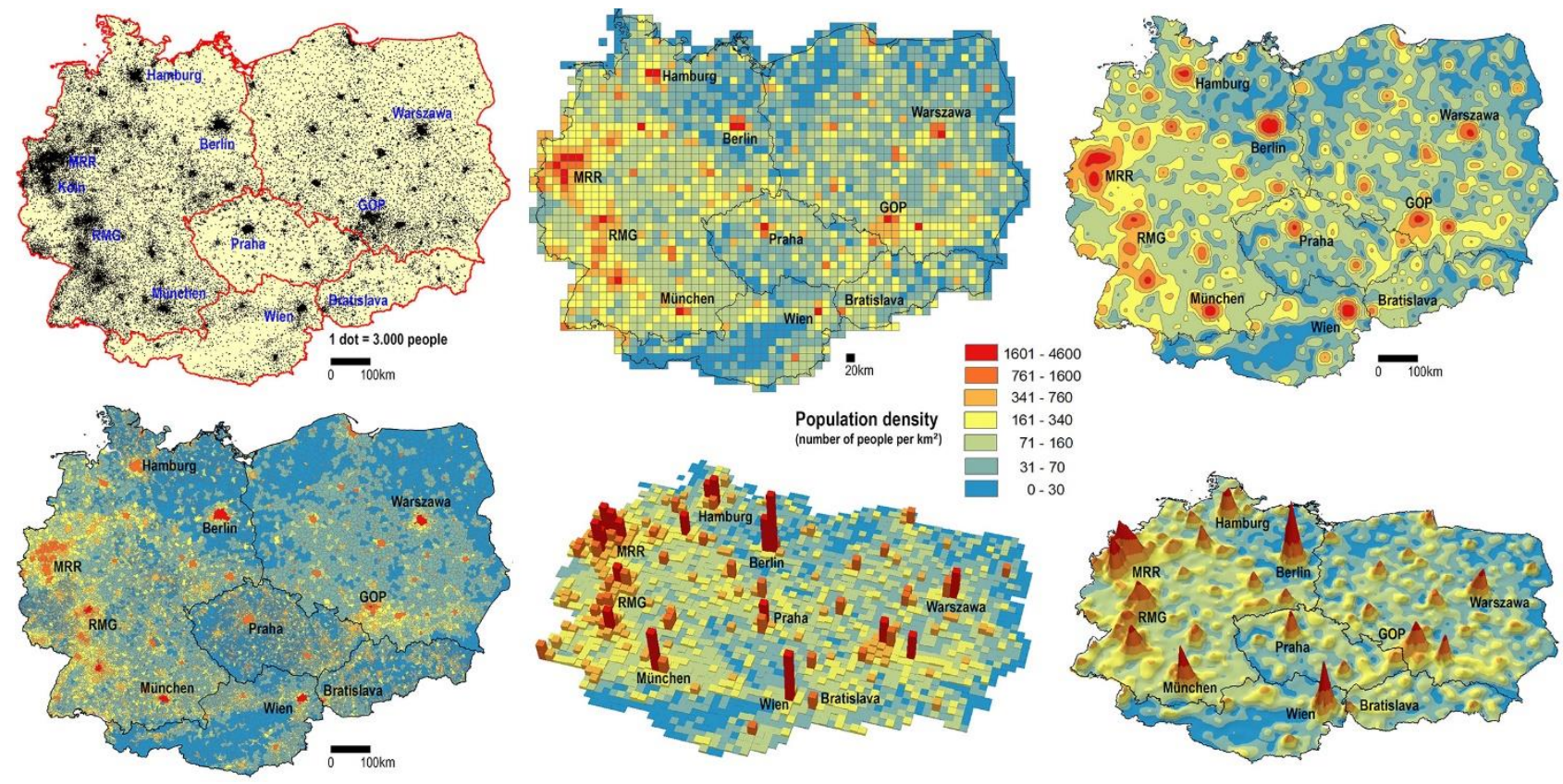

In this study six static representation methods for representing population density are assessed for their effectiveness. Users responses to questions about population distribution are used gather information about the effectiveness of the representation methods. Users were subsequently asked to rank the maps in order of their attractiveness. In a second test, users compared two types of static three-dimensional representations, which included names of key locations with animated versions of the three-dimensions versions without names. As before, information was gathered about the effectiveness of each representation and users asked about the attractiveness of each form of representation. The results of the testing are presented and analysed with a focus on the relationship between the maps efficiency and the users' perceived attractiveness of the method. 